\title{
Croissance et développement du système racinaire de semis de trois espèces de conifères : Pseudotsuga menziesii, Pseudotsuga macrocarpa et Cedrus atlantica
}

\author{
Ph. GRIEU, G. AUSSENAC \\ INRA, Station de Sylviculture et de Production \\ Centre de Recherches de Nancy, Champenoux, F 54280 Seichamps
}

\begin{abstract}
Résumé
Cette étude a pour objectif de comparer la croissance racinaire en minirhizotrons, de trois espèces de conifères, Pseudotsuga menziesii, Pseudotsuga macrocarpa et le Cedrus atlantica, durant les premiers mois de leur existence. Pour ce faire, les allongements et le nombre de racines en croissance ont été mesurés et dénombrés. De plus, ces deux paramètres ont été analysés par tranche de profondeurs de sol.

Les résultats obtenus montrent que pour Pseudotsuga macrocarpa, espèce adaptée aux zones de sécheresses estivales prononcées, le système racinaire atteint plus rapidement les horizons profonds du sol. Par contre, la provenance Ashford de l'espèce Pseudotsuga menziesii, issue des régions côtières de l'ouest de l'Amérique du Nord, présente un développement racinaire moins rapide et moins profond. La troisième espèce étudiée, Cedrus atlantica, originaire des montagnes de l'Afrique du Nord, possède un comportement racinaire intermédiaire.
\end{abstract}

Il apparaitrait donc, sachant que Pseudotsuga macrocarpa ne possède pas une régulation stomatique efficace (GriEu et al., 1988), que cette espèce est adaptée aux régions arides, au moins en partie, grâce au développement rapide et profond de son système racinaire. atlantica.

Mots clés: Croissance racinaire, Pseưdotsuga menziesii, Pseudotsuga macrocarpa, Cedrus

\section{Introduction}

Le fonctionnement des végétaux, et en particulier des arbres forestiers, en situation de sécheresse, dépend en dehors des facteurs du milieu, de différents paramètres qui relèvent de caractéristiques morphologiques, anatomiques, biochimiques et physiologiques. Aussi l'appréciation du degré d'adaptation des espèces ou écotypes au déficit hydrique doit prendre en considération l'ensemble des composantes de l'arbre qui sont concernées par la circulation de l'eau dans le système sol-plante-atmosphère.

Si des travaux relativement nombreux ont porté sur le système racinaire des végétaux des régions arides (OPPENHEIMER, 1961), peu d'études ont été faites sur les arbres forestiers des régions à sécheresses modérées. Très souvent dans ces cas, l'explication de résistances plus ou moins importantes aux déficits hydriques a été recherchée dans les phénomènes de régulation stomatique ou, plus récemment, d'osmorégulation. En relation avec les problèmes de reboisement, la plupart des études sur les 
systèmes racinaires ont concerné les rythmes et les potentialités de régénération des racines. Cependant, Heiner et Lavender (1972) ont montré que le taux de survie de semis en phase de dessèchement dépendait très fortement de leur capacité à produire un système racinaire capable d'explorer les horizons profonds du sol.

C'est en raison des difficultés techniques rencontrées pour effectuer ce type d'étude que l'appréciation de la contribution du système racinaire au fonctionnement (performance) d'ensemble des espèces forestières a été peu abordée, bien que ces recherches soient essentielles pour bien caractériser l'écophysiologie des arbres, notamment à l'égard des problèmes d'adéquation espèce (provenance, écotype) - milieu auxquels sont confrontés les reboiseurs.

La présente étude a été entreprise afin de comparer le développement racinaire, et par là même de préciser l'écophysiologie de trois espèces de conifères d'écologie très différente :

- Pseudotsuga menziesii (Mirb.) Franco var. menziesii Franco (douglas vert), espèce originaire de zones à climat maritime de l'ouest de l'Amérique du Nord.

- Pseudotsuga macrocarpa (Torr.) Mayr., espèce originaire d'une zone de moyenne altitude, à climat aride, du sud de la Californie.

- Cedrus atlantica Manetti (cèdre de l'Atlas), essence originaire des montagnes de l'Afrique du Nord, à climat méditerranéen avec des sécheresses estivales marquées.

\section{Matériels et méthodes}

L'expérimentation a été réalisée dans 9 minirhizotrons ayant les dimensions suivantes : longueur $150 \mathrm{~cm}$, largeur $30 \mathrm{~cm}$, épaisseur $1,5 \mathrm{~cm}$. Ces minirhizotrons ont été remplis d'une terre provenant d'un sol brun ocreux humifère. Ils ont été installés dans des étuis étanches, avec une inclinaison de $45^{\circ}$, dans le sol d'une clairière forestière à proximité du laboratoire.

Dans chaque minirhizotron ont été plantés, côte à côte, un semis de chacune des trois espèces étudiées: Pseudotsuga menziesii (Mirb.) Franco var. menziesii Franco provenance Ashford, Pseudotsuga macrocarpa (Torr.) Mayr, provenance Californie. Cedrus atlantica Manetti provenance Ventoux. Les semis sont âgés de 3 mois. La plantation a été faite en août 1983 en transférant la motte d'origine pour éviter tout risque de crise de transplantation.

Les plants ont été maintenus à la capacité au champ par des arrosages réguliers.

L'étude de la croissance du système racinaire a été effectuée d'octobre 1983 à août 1984. Mensuellement, les minirhizotrons ont été extraits de leurs étuis pour effectuer un suivi du développement racinaire (réalisé sur des films transparents de polyéthylène à l'aide de crayons marqueurs de couleurs différentes). Les paramètres suivants ont été mesurés ou calculés :

- l'allongement du système racinaire :

- allongement longitudinal $(A L)$, somme des allongements de toutes les racines existantes ;

- allongement latéral (Al), somme des allongements des nouvelles racines latérales. 
- le nombre de racines en croissance longitudinales (NL), et le nombre de racines en croissance latérales $(\mathrm{Nl})$.

Les différents allongements et nombres de racines ont été étudiés par tranches de profondeurs de sol (de 10 en $10 \mathrm{~cm}$ ) dans les minirhizotrons. Cette mesure a permis d'avoir une information sur la répartition spatiale (stratigraphie) de la croissance racinaire.

Les données thermiques pour la période d'observation sont présentées dans le tableau I.

TABLEAU 1

Relevé des températures pendant la période d'observation (septembre 1983 - août 1984).

Temperature data during observations (September, 1983 - August, 1984).

\begin{tabular}{|c|c|c|}
\hline & \multicolumn{2}{|c|}{ Température moyenne } \\
\hline & Air (sous abris) & Sol $(-10 \mathrm{~cm})$ \\
\hline Septembre $\ldots \ldots \ldots \ldots \ldots \ldots$ & 15.1 & 11.0 \\
\hline Octobre $\ldots \ldots \ldots \ldots \ldots \ldots$ & 10.4 & 8.6 \\
\hline Novembre $\ldots \ldots \ldots \ldots \ldots \ldots \ldots$ & 4.4 & 2.9 \\
\hline Décembre . . . . . . . . . . . & 2.2 & -0.3 \\
\hline Janvier $\ldots \ldots \ldots \ldots \ldots \ldots$ & 2.6 & -0.3 \\
\hline Février $\ldots \ldots \ldots \ldots \ldots \ldots \ldots \ldots \ldots \ldots \ldots \ldots \ldots$ & 1.7 & -1.4 \\
\hline $\operatorname{Mars} \ldots \ldots \ldots \ldots \ldots \ldots \ldots$ & 4.0 & -0.9 \\
\hline$\ldots \ldots \ldots \ldots \ldots \ldots \ldots \ldots \ldots$ & 8.0 & 3.3 \\
\hline Mai $\ldots \ldots \ldots \ldots \ldots \ldots \ldots$ & 10.5 & 6.9 \\
\hline Juin $\ldots \ldots \ldots \ldots \ldots \ldots \ldots \ldots$ & 14.9 & 11.2 \\
\hline Juillet $\ldots \ldots \ldots \ldots \ldots \ldots \ldots \ldots$ & 17.6 & 13.5 \\
\hline Août $\ldots \ldots \ldots \ldots \ldots \ldots \ldots \ldots \ldots \ldots \ldots \ldots \ldots \ldots$ & 18.1 & 13.5 \\
\hline
\end{tabular}

\section{Résultats}

\subsection{Croissance des systèmes racinaires}

La figure 1 représente l'évolution comparée de la croissance des systèmes racinaires des trois espèces.

Globalement, la croissance racinaire pour les trois espèces présente 2 phases : une première phase au printemps (avril et mai) suivie d'un important ralentissement (mi-mai et juin) puis d'une nouvelle phase de croissance de juillet à novembre. Ces résultats sont en accord avec ceux de Krueger et Trappe (1967) et de Willm (1985) pour le douglas, et de Riedacker (1978) et El Nour (1984) pour le cèdre.

L'examen des différents paramètres mesurés fait apparaître cependant des différences : en ce qui concerne l'allongement des racines préexistantes (AL), les valeurs obtenues sont plus élevées pour $P$. macrocarpa en octobre, novembre, mai, juillet et 
août, que pour les 2 autres espèces (différences très significatives entre $P$. macrocarpa et $P$. menziesii en octobre, novembre, mai et juillet, et significatives en août). Le douglas vert présente la croissance la plus faible. Sa croissance racinaire est nulle entre décembre et mars alors que les deux autres espèces présentent un allongement en février et en mars. L'allongement des racines du cèdre est intermédiaire entre celui des 2 espèces de douglas.

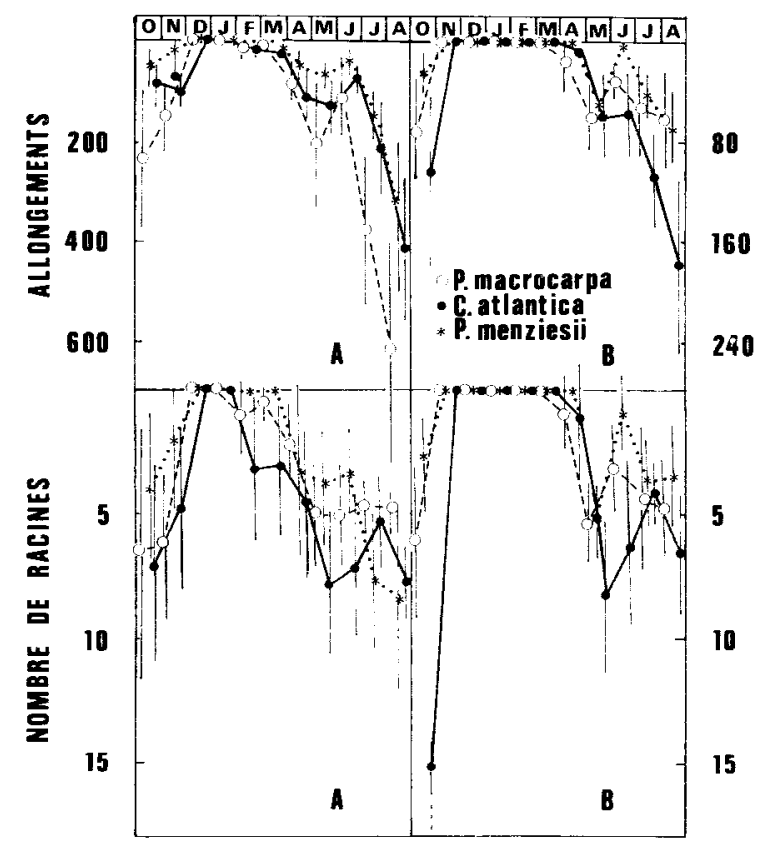

Fig. 1

Evolution mensuelle des allongements et du nombre de racines en croissance (moyennes et écarts-type) pour les trois espèces étudiées.

Monthly evolution of number of roots in growth and root elongation (Averages and standard deviation) for three species studied.
A : racines préexistantes / existing roots.
$\mathrm{B}$ : racines nouvelles / new roots.

La comparaison des allongements des nouvelles racines $(\mathrm{Al})$ ne fait pas apparaitre les mêmes différences : les valeurs obtenues sont voisines pour les deux espèces de douglas, et restent nettement inférieures à celles du cèdre (différences significatives en juillet et très significatives en août).

L'évolution du nombre de racines préexistantes en croissance (NL) est différente entre le douglas vert et le $P$. macrocarpa. Particulièrement en juillet et août, le nombre de racines préexistantes en croissance est nettement plus élevé pour le douglas vert que pour Pseudotsuga macrocarpa. Le cèdre se distingue nettement des deux autres espèces par l'existence d'un nombre important de racines préexistantes en croissance de février à juin, suivi d'une dépression en juillet, puis d'une réaugmentation en août. 
Le nombre de nouvelles racines (Ni) évolue sensiblement de la même manière pour les deux espèces de douglas, avec un maximum en mai. Le cèdre présente aussi un maximum en mai, mais le nombre de nouvelles racines en croissance est beaucoup plus important (différence très significative avec les 2 espèces de douglas).

\subsection{Stratigraphie de la croissance des systèmes racinaires}

L'examen des niveaux de profondeur (strates) dans lesquels une croissance racinaire longitudinale et/ou latérale peut être observée révèle un certain nombre de faits significatifs (fig. 2).

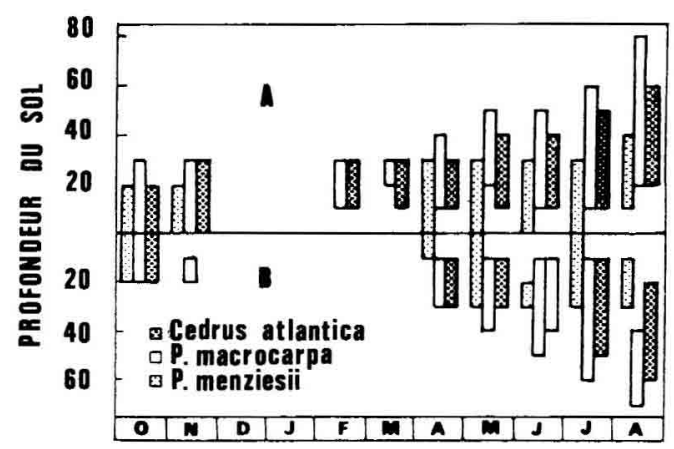

FIG. 2

Profondeurs de sol dans lesquelles a été observée mensuellement une croissance racinaire pow les trós espèces t́tudiées.

Soil depth at which monthly root growth of tree species was observed.

A : croissance des racines préexistantes / growth of existing roots.

$\mathrm{B}$ : croissance des racines nouvelles / growth of new roots.

En particulier, il apparait que c'est $P$. macrocarpa qui prospecte le plus rapidement les horizons prodonds du sol. Son enracinement est déjà, dès le mois d'octobre, dans la tranche des 20 à $30 \mathrm{~cm}$ de profondeur, et en août, il atteint la tranche des 70 à $80 \mathrm{~cm}$. En quatre mois de végétation, le système racinaire de cette espèce a progressé de $50 \mathrm{~cm}$.

Pseudotsuga menziesii explore le sol nettement moins rapidement, aussi bien en automne après plantation, que pendant la saison de végétation. En fin d'étude (août), son système racinaire n'atteint que $30-40 \mathrm{~cm}$ de profondeur.

Le cèdre est intermédiaire entre les deux espèces précédentes. Son système racinaire atteint la tranche de $20-30 \mathrm{~cm}$ de profondeur en automne, et la tranche des $50-60 \mathrm{~cm}$ en août.

Ainsi, comparé à Pseudotsuga menziesii et Cedrus atlantica, Pseudotsuga macrocarpa est caractérisé par une plus grande aptitude à explorer les couches les plus profondes du sol. 


\section{Discussion}

Les résultats obtenus dans cette étude font apparaître des différences fondamentales dans les cinétiques de croissance et dans les stratégies d'occupation des sols par les racines des 3 espèces comparées.

Pseudotsuga macrocarpa se caractérise par une croissance racinaire très rapide permettant une exploration des horizons profonds du sol. Au contraire, Pseudotsuga menziesii a un enracinement moins rapide, la croissance des nouvelles racines représente une part plus importante de l'allongement racinaire total.

Cedrus atlantica occupe une position intermédiaire entre ces deux espèces.

Si l'on se réfère maintenant aux informations disponibles sur l'écologie de ces trois espèces, on constate que :

- Pseudotsuga macrocarpa, espèce se développant naturellement dans des conditions climatiques de sécheresses estivales importantes, n'a pas, sur le plan de la régulation des échanges gazeux, un comportement particulièrement performant (GRIEU et al., 1988). Il en résulte qu'il semble que ce soit dans cette spécificité de son développement racinaire, qui lui permet d'atteindre rapidement les horizons profonds du sol mieux alimentés en eau, que réside le niveau d'adaptation écophysiologique de cette espèce.

- Pseudotsuga menziesii var. Ashford, espèce et provenance se développant dans des régions à climat plus humide où l'existence de sécheresses estivales même modérées est peu fréquente en comparaison des stations où pousse l'espèce précédente, possède un développement racinaire beaucoup moins performant. Ce phénomène pourrait être mis en relation avec le fait que le système racinaire du douglas vert n'ait atteint qu'une profondeur de $15 \mathrm{~cm}$ contre $25 \mathrm{~cm}$ pour les 2 autres espèces. En effet, en janvier, février et mars, le sol dans les 15 premiers $\mathrm{cm}$ était à une température voisine probable de $0{ }^{\circ} \mathrm{C}$ (tabl. 1), alors qu'en profondeur, la température était plus élevée. On sait en effet que les seuils de températures pour la croissance racinaire se situent entre $2^{\circ} \mathrm{C}$ et $4^{\circ} \mathrm{C}$ (Lavender \& Overton, 1972 ; Riedacker, 1976). Cependant, lorsqu'au printemps la température du sol remonte, la croissance racinaire de $P$. menziesii reste toujours inférieure à celle des deux autres. Il semble alors que l'essentiel des possibilités de résistance à d'éventuels déficits hydriques de cette provenance de douglas vert consiste dans un système efficace de régulation stomatique (Grieu et al., 1988).

- Cedrus atlantica, espèce se développant dans des conditions de sécheresse très marquées des zones méditerranéennes, a une croissance racinaire qui se situe en position intermédiaire par rapport aux deux espèces de douglas. Ses capacités de régulation stomatique sont faibles comparées à celles de Pseudotsuga menziesii et quasiéquivalentes à celles de Pseudotsuga macrocarpa (Grieu et al., 1988). Au moins dans les premiers mois de la vie de l'arbre, son système racinaire pénètre moins vite en profondeur dans le sol que celui de Pseudotsuga macrocarpa, et il semble sur ce plan moins performant.

Ces résultats soulignent toute l'importance de la compréhension et de la détermination précises des composantes du fonctionnement de l'arbre, en particulier de la composante racinaire, pour expliquer la réponse et l'adaptation des espèces aux phénomènes de sécheresse. D'un point de vue sylvicole, il convient aussi de remarquer que l'utilisation des espèces, qui, comme Pseudotsuga macrocarpa, tirent leurs performances 
du développement de leur système racinaire, doit être envisagée avec précaution ; leur introduction sur des sols superficiels, sans possibilité de pénétration des racines, se solderait par des échecs. A cet égard, il faut souligner tout l'intérêt des techniques de préparation du sol, notamment du soussolage en zone méditerranéenne.

Reçu le 25 mai 1987.

Accepté le 24 novembre 1987.

\begin{abstract}
Summary
Growth and development of root system in three conifer species : Pseudotsuga menziesii, Pseudotsuga macrocarpa and Cedrus atlantica
\end{abstract}

This study was aimed at comparing root growth in minirhizotrons of three conifers : Pseudotsuga menziesii, Pseudotsuga macrocarpa and Cedrus atlantica during their first few months of existance. Root length and number of roots in growth have been studied. Moreover, these two parameters have been analysed. with depth of soil profile.

Résults obtained show that for Pseudotsuga macrocarpa, species adapted to the zones of high summer drought, the root system reaches rapidly in deep soil horizons, as against the ASHFORD provenance of Pseudotsuga menziesii, indigeneous to west coastal regions of North America, which presents slow root development having shallow depths. Third species studied, Cedrus atlantica, coming from the mountains of North Africa presents an intermediate root system.

It appears, therefore, that Pseudotsuga macrocarpa is adapted to arid regions, at least partially, because of its deep and rapid root system development as it does not possess an efficient stomatal regulation (Grieu et al., 1988).

Key words : root growth, Pseudotsuga menzicsii, Pseudotsuga macrocarpa, Cedrus atlantica.

\title{
Références bibliographiques
}

EL Nour M., 1984. Etude de la croissance et de la régénération des racines du chêne pédonculé, du cèdre, du pin laricio de Corse et du pin noir. Essais d'amélioration de la reprise après plantation à partir de modifications du fonctionnement racinaire. Thèse Nancy I et INRA, $118 \mathrm{p}$.

Grieu P., Guehl J.M., Aussenac G., 1988. The effect of soil and atmospheric drought on photosynthesis and stomatal control of gas exchange in three coniferous species. Physiol. Plant. (In press).

Heiner T.Ch. \& Lavender D.P., 1972. Early growth and drought avoidance in douglas fir seedlings. Research paper 14,7. Forest research laboratory School of forestry O.S.U. Corvailis.

Krutger K.W. \& Trappe J.M., 1967. Food reserve and seasonnal growth of douglas fir seedlings. For. Sci., 13, 192-202.

LAVENDER D.P. \& OVERTon W.S., 1972. Thermoperiods and soil temperature as they affect growth dormancy of douglas fir seedlings of different geographic origines. Research paper 13. Forest research laboratory O.S.U. Corvalis.

Oppenheimer H.R., 1962. L'adaptation à la sécheresse. Le xérophytisme. Recherches sur la zone aride ou semis aride. U.N.E.S.C.O., 115-153.

RiedACKer A., 1976. Rythmes de croissance et de régénération des racines des végétaux ligneux. Ann. Sci. For., 33 (3), 109-138.

RiEDACKER A., 1978. Régénération et croissance de la partie souterraine et aérienne de cèdres placés sous climat constant. Ann. Sci. For, 35 (2), 117-138.

WiLlm F., 1985. Etude de la phénologie et de la croissance pondérale des parties aériennes et souterraines de jeunes plants de douglas et de cèdre placés dans différentes conditions climatiques. D.E.S. Sciences naturelles, Nancy I, 24 p. 